\title{
New developments in particle-based immunoassays: introduction
}

\author{
Leigh B. Bangs \\ Bangs Laboratories Inc., 979 Keystone Way, Carmel, IN 46032-2823 USA
}

\begin{abstract}
There have been many innovations in diagnostics since white latex particles or microspheres were first used in medical diagnostic applications as "latex" agglutination tests (LAT) in the late 1950's. These innovations include colored particles permitting multivalent (or simultaneous) analyses, and special devices for simplifying test execution and result interpretation. Dyed agglutinated particles caught on filters form the basis of another class of tests. Sensitive particle-enhanced turbidimetric assays are in common use and are read with clinical chemistry analyzers via spectrophotometric or nephelometric methods. Particle capture ELISA tests and assays are in common use. The popular new strip tests for pregnancy, ovulation, drugs of abuse in urine, and many other tests all use dyed microspheres (and some use two types of microspheres). Solid phase assays and tests use particles as a solid phase for positive or negative capture of a wide variety of analytes. Solid-liquid separations can be made by centrifugal density separation, or filtration, or via magnetic separation of superparamagnetic particles. Single microsphere (and perhaps single molecule sensitivity?) assays are now possible in flow cytometers.
\end{abstract}

\section{Introduction}

Microsphere-based diagnostic tests (qualitative, yes/no results) and assays (quantitative results) are usually based upon the very specific interaction of antigen $(\mathrm{Ag})$ and antibody $(\mathrm{Ab})$. Sub-micron sized polystyrene (PS) microspheres, often called "uniform latex particles", are used for the solid support; Ab or Ag can be adsorbed onto them. These "sensitized" microspheres then act to magnify or amplify the Ag-Ab reaction which takes place when they are mixed with a sample containing the opposite reactant. In simple particle agglutination, a positive test results when uniformly dispersed milky-appearing Ab-coated particles, in a drop of water on a glass slide, react with $\mathrm{Ag}$ in a drop of sample (whole blood, serum, urine, etc.) to cause agglutination or clumping of the microspheres, to look like curdled milk. Alternatively, Ag-coated particles are agglutinated by a positive sample of $\mathrm{Ab}$ (Fig. 1).

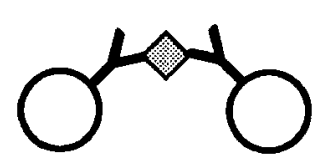

Tests

Latex Agglutination Tests

(Slide, Tube or 96-well Plate)
Assays

Turbidimetric Immunoassays

Nephelometric Immunoassays

Where<smiles>C1CCCCCC1</smiles>

$=$ MicroSphere,

$\mathcal{L}=$ Antibody $(\mathrm{Ab})$, and

$\varnothing=$ Antigen $(\mathrm{Ag})$

Fig. 1 Agglutination tests and assays.

\section{Qualitative Agglutination (Tests)}

Microsphere or latex agglutination tests (LAT's), first used in 1956 for a rheumatoid factor test (1), have been applied to $>100$ infectious diseases. There are also LAT's for $>60$ other chemical analytes, e.g., hCG, RF, CRP, ASO, FDP, and fecal occult blood. There have also been many other applications in veterinary medicine, plant health, law enforcement, food, and the environment. 
Tests for new analytes are continually being added, such as an LAT for lactoferrin released from leukocytes in diarrheal stool specimens- a screening test for "traveller's diarrhea" (2). A recent occult blood test is actually a dual LAT-for hemoglobin and transferrin in feces for "... a much higher detection rate compared to hemoglobin [alone]". Also new is an LAT for BFP in urine, a new tumor marker for bladder cancer (3).

LAT's are portable, useful everywhere, rapid, and efficient. Ideal for point-of-care uses (field, ambulance, or bedside), they can be run quickly and simply ( 2 minutes from sample preparation). Diagnosis and treatment can commence promptly, before the patient is transferred or discharged (4).

\section{Slide Test Improvements}

The earliest "ordinary" slide tests used plain, white microspheres on washable, reusable glass slides, usually with a black background. Recent refinements include novel devices designed to make the tests less technique-dependent. In Roche's OnTrak ${ }^{\mathrm{TM}}$ device the sample and coated microspheres are mixed and guided into a "track" or capillary. As the reactants move down the track by capillary action, they mix themselves, and agglutination is read with transmitted light after they reach the end 2-3 minutes later. No hand rocking or rotation is necessary, and the test is quite "hands off" and operator-independent.

Several companies produce tests with reagent coated microspheres dried on a card. In use, the microspheres are rehydrated with sample, stirred, and observed for agglutination. Dried reagents offer excellent shelf-life.

Dyed microspheres provide different contrast (dyed microspheres observed against a white background). Some Staphylococcus tests use red or black microspheres, and there is a new black LAT for $E$. coli 0157:H7 (the strain implicated in food-poisoning due to under-cooked meat). Soon to appear are two or three LATs for $M$. tuberculosis, et al. using dyed microspheres.

MUREX has a Salmonella test which uses antibodies to three different antigen groups bound to three different colored microspheres (red, blue, and green). By comparing the color of the combined agglutinated microspheres to a background color, one can decide which salmonella groups are present in the sample. They also have a Shigella test kit employing two colors of microspheres and two reagents, to identify a total of 4 different strains.

Now RPLA (reverse passive latex agglutination) tests use more stable dyed microspheres (latex particles) to replace the red blood cells in hemagglutination tests (5).

\section{Quantitative Agglutination (Assays): Spectrophotometry/Nephelometry}

Spectrophotometers and nephelometers have been used for years to measure direct protein precipitation. When these instruments, which measure absorbed, or scattered light, are used in place of the human eye, it is possible to quantitate agglutination and to develop very sensitive microsphere agglutination immunoassays. Many clinical chemistry analyzers now feature some turbidimetric (spectrophotometric) "latex" assays. Behring's "latex-enhanced" nephelometric method was proposed as the reference method for C-reactive protein (CRP) (6). New assays continue to appear: Dako AS (Denmark) offers a cystatin C assay- described as a "better marker than serum creatinine for glomerular filtration rate" (7). Orion Diagnostica (Finland) has a new very compact and sophisticated "doctor's office" size turbidimeter, and they are steadily adding new microsphere assays.

\section{Other Instrumental Agglutination Methods}

Sensitivity 10-15 times better than turbidimetry has been claimed using particle counters to measure changes in numbers of single particles or clumps of particles during agglutination (8). Sienna Biotech's new Copalis ${ }^{\mathrm{TM}}$ technology counts single, Ab-coated $\sim 1 \mu \mathrm{m}$ microspheres before and after agglutination. Using "optical sizing flow particle analysis" (sensitive laser-based particle sizer/counter), they measure the decrease in single particles as proportional to analyte concentration. They can do several tests simultaneously using different sized microspheres, coated with different Ab's. They can use whole blood (no interference from cells), and claim sensitivity for $\mathrm{TSH}<1 \mathrm{pM}$.

With "Spherotest", Indicia (Oullins, France) has made agglutination colorimetric! Dispersions of undyed microspheres of diameter, $\mathrm{d}$, dispersed in a liquid with closely matched refractive index will appear colored. They will absorb a maximum amount of light of wavelength $\lambda_{\max } \approx d / 2$. Thus, 740nm microspheres (dispersed in appropriate liquid) absorb at $\lambda_{\max }=340 \mathrm{~nm}$ (blue) and appear yellow. Upon agglutination, clumped particles appear as a different color and loss of single microspheres results in a drop of absorbance at $\lambda_{\max }\left[\mathrm{A}\left(\lambda_{\max }\right)\right]$. Then, a plot of $\mathrm{A}\left(\lambda_{\max }\right)$ vs. analyte concentration follows only the single particle concentration (ignoring agglutinated particles) and can yield a linear curve over 5 orders of magnitude! (9).

Magnetic microsphere agglutination can be measured by magnetic moment analysis (10) and agglutination of fluorescent dyed microspheres could be studiedwith fluorescence quenching analysis. 
Filter Separation Agglutination Tests (\& Assays)

Kodak's earliest Surecell test kits used dyed agglutinated microspheres caught on a filter. Red microspheres coated with $\mathrm{Ab}$ were incubated with a sample and poured onto a filter. Single microspheres passed through the filter and no color appeared on the surface. If the sample contained the appropriate $\mathrm{Ag}$, the microspheres agglutinated, and the agglutinated clumps were caught upon the filter resulting in a red (or pink) positive color test for the $\mathrm{Ag}$ (Fig. 2).

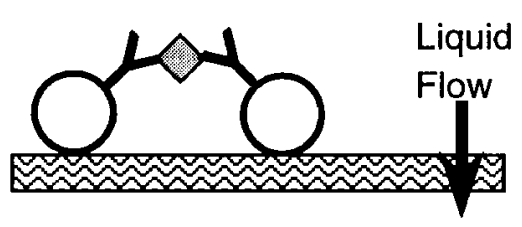

Tests

Agglutination or

Coagglutination and Capture by Filter
Assays

(Quantifiable with reflectometer or strip reader.)

Fig. 2 Filter separation agglutination tests (\& assays)

Carter-Wallace's home pregnancy test, First Response ${ }^{\circledR}$, uses plain microspheres $(\sim 1 \mu \mathrm{m})$ coated with one antibody to hCG and very small $(<50 \mathrm{~nm})$ red gold sol particles coated with antibody to another hCG epitope. On mixing with a sample of urine, if the sample contains hCG, the particles are coagglutinated, yielding red clumps. The mixture is poured through a filter which catches the red clumps to yield a pink colored filter. With negative urine, unagglutinated red particles pass through the filter and no color develops on it.

The principles employed in these two tests could easily be applied to assays where the reflected color intensity (as measured perhaps in a dry strip reader?) would correlate with the sample's Ag content. Such assays would be comparable to the ELISA's below, but would be simpler, and probably more stable (no enzymes).

\section{Particle Capture Enzyme Linked ImmunoSorbent Tests and Assays}

To prepare these tests: $\mathrm{Ab}$ is bound to microspheres; the microspheres are caught on a filter and dried (Fig. 3). When a sample is passed through the filter, $\mathrm{Ag}$ is caught by the $\mathrm{Ab}$ on the microspheres. Then, a second $\mathrm{Ab}$-enzyme conjugate is put through the filter and is caught by the $\mathrm{Ag}-\mathrm{Ab}$ on the microspheres to complete the sandwich. Finally, when enzyme substrate is passed through the filter, the enzyme reacts with substrate to create an insoluble colored product on the filter surface which is proportional to the amount of $\mathrm{Ag}$ caught.

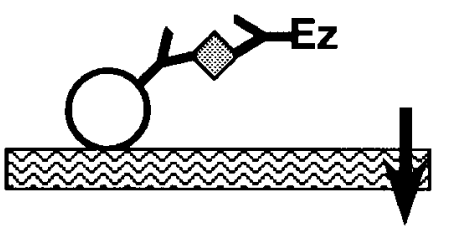

Tests

Assays

Particle Capture ELIST

Particle Capture ELISA

Fig. 3 Particle-capture Enzyme-Linked ImmunoSorbent Tests and Assays

Various tests (like hCG, Strep A, and others) using this principle have been made by Hybritech (ICON), Abbott (Test Pack), Novo Nordisk (11), IDEXX, and others. Assays have been made by Hybritech (ICON QSR), Abbott (IMX, and AxSym), Neogen (Reveal), and others. Using a reflectance meter, the colored spots caused by analyte are compared to a blank and a standard spot to yield a true quantitative assay. Reveal is literally a "field" instrument (pocket-sized) for measuring plant pests like fungal infestations of soybeans, and turf diseases on golf courses.

Dyed Microsphere Sandwich Test (also called "One Step", Immunochromatographic, or Strip Tests) In 1988 a new over-the-counter pregnancy test (Clearblue Easy®, developed by Unipath) revolutionized diagnostic immunological tests. The test uses dyed microspheres in a sandwich format to give a one step test eliminating the need for color-generating enzymes (Fig. 4) (12). Now many other companies offer similar tests for hCG, "popular" infectious diseases, and drugs of abuse in urine (DAU) (Table 1). Triage ${ }^{\mathrm{TM}}$ from Biosite Diagnostics is an eight analyte DAU inhibition test panel $(13,14)$. 


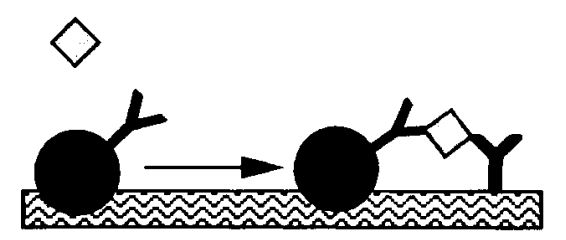

Fig. 4 "One Step" strip or chromatographic tests and assays. First antibody-coated, dyed microspheres migrate along strip to immobilized second antibody; antigen sandwich forms colored line.

All of these tests also have the promise of becoming true assays. If the intensity of color formed could be read by a dry strip reader, for example, then a quantitative result could be obtained. Different colors of dyed microspheres could be used and different analytes color coded.

Solid Phase Assays (requiring solid/liquid separation)

Microspheres have unique properties - small enough to remain suspended for hours or longer at normal gravity, yet easily separated from suspension with a centrifuge, magnet, or filter. They have been used for years as solid supports for radioimmunoassays and other newer assays where solid/liquid (bound/unbound) separation is needed (Fig. 5).

In Genzyme's Direct LDL [Low Density Lipoprotein] Cholesterol Immunoseparation Reagent Kit, microspheres coated with $\mathrm{Ab}$ (goat anti-human apolipoproteins) are mixed with serum or plasma and incubated in a separation device. After centrifuging the device to filter the microspheres from the liquid, LDL cholesterol in the filtrate is measured using conventional enzymatic cholesterol reagent.
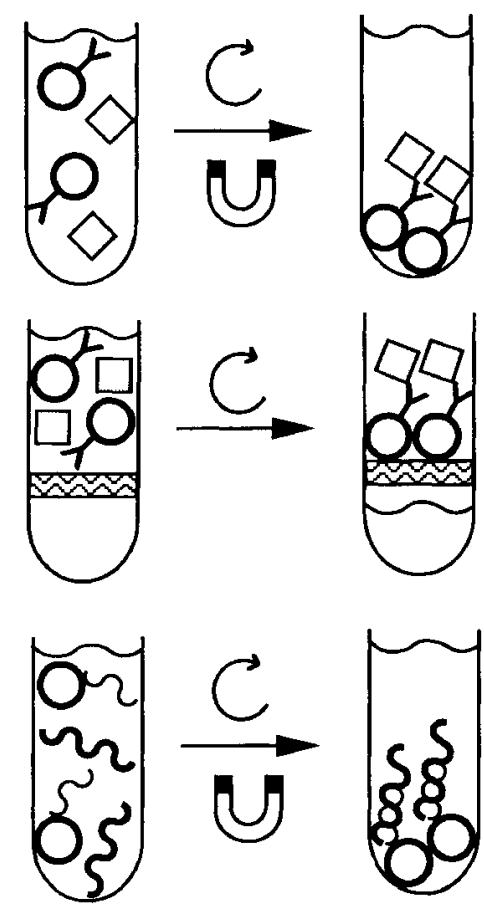

\section{Assays}

Solid Phase Immunoassays with Centrifugal or Magnetic Separation (includes cell separation or concentration)

\section{Solid Phase Immunoassays with Filtration Separation}

DNA/RNA Adsorption onto Silica
MicroSpheres or Oligo DT
Covalently Bound to Magnetic or
Silica Particles which Captures
Poly A Tail of DNA Probe or mRNA

Fig. 5 Solid phase assays (requiring solid/liquid separation)

Superparamagnetic Particle Based Assays: "Magnetic" particles permit fast and easy separation of solid and liquid phases. Actually superparamagnetic, the particles respond to a magnet but they retain no residual magnetism after removal of the magnet. They can be used to help pull things out of solution more quickly, as in commercial solid phase RIA's, ELISA's, and newer chemiluminescent assays by Amersham, Ciba-Corning, and Biotrol (15). Immunoradiometric assays (IRMA's) can also be done with magnetic particles (16). Sanofi's ACCESS instrument uses magnetic microspheres with chemiluminescent assays such as the one for ferritin $(17,18)$. 
In the human genome project, the microspheres are used in the first step of rapid DNA purification (19). Promega's PolyATtract mRNA isolation procedure uses biotinylated oligo-dT hybridized with mRNA and bound to streptavidin-coated magnetic particles. After magnetic separation, the mRNA can be eluted from the magnetic beads for recovery (20).

Other molecular biology applications of magnetic microspheres include oligonucleotide purification (21), "rapid genomic walking" (22), and sequencing (23). In the Wilson paper, uncoated magnetic particles are used twice to purify ss-DNA- first to collect aggregated M13 phage and later to collect its DNA from ethanol. Magnetic particles are cited as relatively inexpensive raw materials in a method which reduces labor cost by half. In another DNA hybrid assay (by IGEN), streptavidin-coated magnetic particles are used in a human papilloma virus assay based on PCR (polymerase chain reaction), and read by electrochemiluminescence (24).

Magnetic particles assist in positive and negative cell depletion (cell capture and removal) of bacteria (collecting and concentrating Chlamydia trachomatis from urine for analysis), animal and plant cells, and plant protoplasts $(25,26,27,28)$.

\section{Microspheres as Markers and Stains}

$\mathrm{Ab}$-coated dyed microspheres will stick to cells and identify them. Dyed $\sim 5 \mu \mathrm{m}$ microspheres, with appropriate Ab coatings, have been used as cell tags in "rosette" type tests, where the microspheres cluster around certain cells to identify them in the microscope. The new technique called FISH (fluorescent in situ hybridization) involves labelling of intact cells using fluorescent microspheres.

Zynaxis has a new assay method for enumeration of CD4+ and CD8+ T-lymphocytes using McAb-coated fluorescent dyed microspheres and McAb-coated magnetic microspheres. The mixed microspheres form rosettes around the appropriate T-cells. A magnet separates rosettes from unrosetted cells and permits quantitation of fluorescence (29).

Other applications for dyed microspheres include regional blood flow studies in animals. Multicolored 10 and $15 \mu \mathrm{m}$ microspheres, injected into an animal's circulatory system, become lodged in the tissues during circulation. After tissue biopsies, the colored or fluorescent spheres are recovered and quantitated or analyzed for size and fluorescence intensity in flow cytometry instrumentation. They are replacing radiolabelled microspheres in this field $(30,31)$.

\section{Scintillation Proximity Assays}

Amersham has an assay system where one microsphere is coated with a $\gamma$-emitter radio-labelled $\mathrm{Ag}$ and another microsphere is dyed with scintillator and coated with Ab. When they are mixed together, an Ag-Ab reaction binds the microspheres together and light will be given off when $\gamma$-rays emitted from the Ag-coated microspheres enter the Ab-coated, scintillator-dyed microspheres. Any free antigen in a sample added to the mixture will interfere with the two microspheres coming together and decrease light output. (Fig. 6) (32).

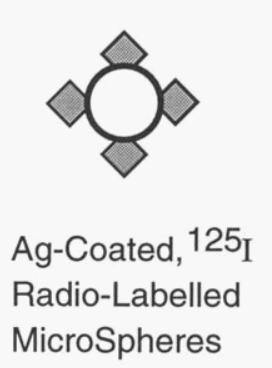

Fig. 6 Scintillation proximity assay. If $\mathrm{Ag} / \mathrm{Ab}$ reaction binds particles together, light will be given off when $\gamma$-rays emitted from Ag-coated microspheres enter $\mathrm{Ab}$-coated, scintillator-dyed microspheres. Free Ag in sample interferes with the two microspheres coming together and decreases light output. 
Some researchers predict that many immunoassays will soon be done with cells and single microspheres in flow cytometry instruments as use of these instruments becomes more widespread $(33,34)$. Antibody-coated microspheres are mixed with sample. If antigen is present the laser light is scattered (or the microsphere fluoresces) differently. The difference in light scattered (emitted) by microspheres with and without Ag will be used to quantify the antigen.

Agglutination tests using silica microspheres will yield different kinds of tests and assays because the $\mathrm{SiO}_{2}$ beads are hydrophilic and have higher density $(\sim 2 \mathrm{~g} / \mathrm{ml})$ than the commonly used polystyrene.

Darkly-dyed microspheres should permit whole blood tests. Black microspheres should be visible even in the presence of red blood cells, unless the RBC's interfere somehow with the agglutination.

"Clear to cloudy" test: Diluted $(\sim 1 \%)$ suspensions of very small $(<50 \mathrm{~nm})$ microspheres are transparent; after agglutination to clumps $>300 \mathrm{~nm}$, the suspension becomes turbid. This could lead to a simple screening test wherein a change from clear to cloudy signals a positive result when sample is added to appropriately-coated microspheres.

The field of biosensors has been "promising" for several years. Perhaps microspheres can help as amplifiers of the signal in the new evanescent-wave-based sensor technology (Fig. 7). Ideally, on an antibody-coated

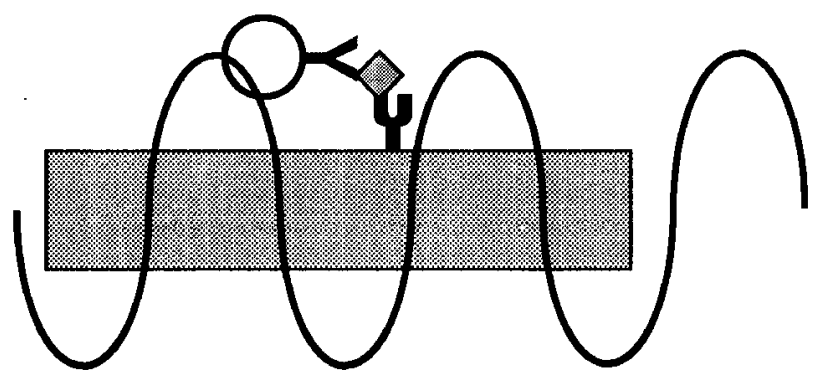

Fig. 7 Possible use of microspheres in biosensors (evanescent wave concept shown). Antibody-labelled microspheres sticking to biosensor via antigen sandwich affect light in optic fiber.

biosensor, if antigen in a sample is bound by the antibody, there will be a detectable signal change. However, if the signal is not strong enough, one can add a second antibody, perhaps with a microsphere attached. Then, there should be a large change in the signal, with amplification brought about by the microspheres.

This is only the beginning! Many more exciting applications of microspheres will surely follow.

\section{References}

1. J.M. Singer, C.M. Plotz, Am. J. Med., 21, 888 (1956).

2. D.M. Lyerly, P. Hahn, American Clinical Lab, 13/5, 18 (1994).

3. M. Ishii, Pharma Japan, October 31,1994.

4. J. Carney, Anal. Proc., 27, 99-100 (1990).

5. H. Fujikawa, H. Igarashi, Appl. Envir. Microbiol., 54/10, 2345-2348 (1988).

6. B.A. Harris, C.E. Hart, and D.A. Nealon, Annales de Biologie Clinique, 51/3,4,5, 484 (1993).

7. J. Kyhse-Andersen, C. Schmidt, G. Nordin, et al., Clin. Chem., 40/10, 1921-1926 (1994).

8. T.A. Wilkins, G. Brouwers, J.-C. Mareschal, et al., Clin. Chem., 34/9, 1749-1752 (1988).

9. S. Legastalois, U.S.Pat. 5,043,289 (1992).

10. K. Imai, D. Tokinaga, and K. Yokogawa, U.S.Pat. 4,913,883 (1990).

11. H. Christensen, H.H. Thyssen, O. Schebye, et al.,Clin. Chem., 36/9, 1686-1688 (1990).

12. Anonymous, Financial Times (London), July 7, 1988; Technology Section.

13. Anonymous, Clin. Lab. Prod. 21/3, 8-9 (1992).

14. K. Buechler, G.E. Valkirs, and R. R. Anderson, U.S.Pat. 5,028,535 (1991).

15. C. Freier, B. Kan, and T. Gicquel, J. Clin. Immunoassay, 14/2, 111-114 (1991).

16. C. Larue, C. Calzolari, J. Léger, et al., Clin. Chem., 37/1, 78-82 (1991).

17. W. Patterson, P. Werness, W.J. Payne, et al., Clin. Chem., 40/11, 2042-2045 (1994).

18. T. Peterson, K. Kapsner, B. Liljander, et al., Clin. Chem., 38/6, 1080 (1992).

19. H. Goldner, $R \& D$ Magazine, 36/4, 32-33 (1994).

20. C. Smith, S. Ekenberg, and M. McCormick, Promega Notes, \#25/June (1990). 
21. G. Fry, E. Lachenmeier, E. Mayrand, et al., BioTechniques, 13/1, 124-131 (1992).

22. D. Warshawsky, L. Miller, BioTechniques, 16/5, 792-798 (1994).

23. R.K. Wilson, BioTechniques, 15/3 414-422 (1993).

24. J. Kenten, J. Casadei, J. Link, et al., Clin. Chem., 37/9, 1626-1632 (1991).

25. A. Hedrum, J. Lundeberg, C. Păhlson, et al., PCR Methods and Applications, 2, 167-171 (1992).

26. J.T. Kemshead, J. Hematotherapy, 1, 35-44 (1992).

27. F. George, Thrombosis and Haemostasis, 67/1 (1992).

28. I. Dörr, S. Miltenyi, F. Salamiini, et al.,H. Uhrig, Bio/Technology, 12, 511-515 (1994).

29. B.D. Jensen, F.A. Vella, M.L. Harner, et al., Clin. Chem., 40/6, 1026 (1994)

30. P. Kowallik, R. Schulz, B.D. Guth, et al.,Circulation, 83/3, 974-982 (1991).

31. R.W. Glenny, S. Bernard, and M. Brinkley, J. Appl. Physiol., 74(5), 2585-2597 (1993).

32. K. Takeuchi, Laboratory Practice, Sept., 1992.

33. T.M. McHugh, Immunochemica, 5/1, 1-6, (1991).

34. Cook, L, Irving, D, J. Clin. Immunoassay, 12/1, 36-39 (1989). 